\title{
Valutazione della capacità di rientro alla base di un elicottero in presenza di danno balistico ad un albero di trasmissione della linea rotore di coda
}

\author{
L. Giudici, A. Manes, M. Giglio \\ Politecnico di Milano, Dipartimento di Meccanica,via La Masa 1 - 20156 Milano, \\ andrea.manes@polimi.it
}

RiAssunTO. Nella progettazione di un elicottero militare, destinato ad operare a bassa quota e in ambiente ostile, il danneggiamento di componenti critici, conseguente ad impatto balistico, riveste un ruolo primario nella valutazione delle possibilità di sopravvivenza dell'intera macchina. In questo articolo è quindi proposto uno studio sperimentale, suddiviso in diverse fasi, riguardante la verifica della capacità di un elicottero di portare a termine una missione di rientro alla base a potenza ridotta e in presenza di danneggiamento balistico ad un albero di trasmissione della linea rotore di coda.

Il lavoro ha richiesto dapprima l'esecuzione, su esemplari del componente in esame, di prove sperimentali di impatto balistico, condotte utilizzando un proiettile calibro 7.62 NATO. Successivamente su ciascun albero danneggiato sono state eseguite prove torsionali statiche ed a fatica, il cui scopo è stato verificare la resistenza residua del componente all'applicazione di opportuni carichi rappresentativi delle sollecitazioni riscontrate durante la missione di rientro.

ABSTRACT. In the design of a military helicopter, with the aim to operate at low altitude into enemy territory, the damage of critical components, caused by ballistic impact, plays a primary role in assessing the survival of the entire machine. In this work an experimental study is proposed, concerning the capability of a helicopter to carry out a mission of return to the base at reduced power, in the presence of a tail rotor shaft damaged by ballistic impact of 7.62 NATO projectile.

In the first part of this study, some specimen representatives of the tail rotor shaft are subjected to experimental ballistic impact tests. Subsequently, static and dynamic torsional tests have been performed on the damaged components in order to asses the residual strength under the loads encountered during the mission.

Parole Chiave. Elicottero, albero di trasmissione, impatto balistico, proiettile.

\section{INTRODUZIONE}

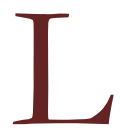

o studio sperimentale qui descritto si articola in due parti: inizialmente sugli alberi di trasmissione disponibili sono state condotte delle prove di impatto balistico, utilizzando un proiettile $7.62 \mathrm{NATO}$; in seguito sono state effettuate delle prove di torsione, sia statiche che di fatica, con l'obiettivo di verificare la resistenza degli alberi danneggiati sotto l'azione delle sollecitazioni derivanti dalla missione.

Tale studio sperimentale rientra nel programma di valutazione del requisito balistico di un componente elicotteristico. Il requisito balistico qui considerato, prevede appunto che, in seguito al danneggiamento di un albero di trasmissione della linea rotore di coda, l'elicottero, benché subisca una parziale riduzione delle sue capacità di manovra, sia in grado di portare a termine una missione di rientro alla base "a potenza ridotta" della durata di circa 30 minuti. 


\section{Prove SPERIMENTALI Di IMPATTO BALISTICO}

\section{Descrizione dell'albero}

$\mathrm{M}$

entre diversi esperimenti di impatto sono stati condotti su lastre di vari materiali e spessori, sono pochi gli studi pubblicati riguardanti tubi metallici. In [1] e [2] è contenuta una descrizione dettagliata di una vasta gamma di impatti, e sono enunciati alcuni concetti fondamentali di balistica utili alla comprensione del fenomeno.

L'albero di trasmissione oggetto di studio è costituito da un tubo cilindrico cavo a parete sottile ai cui estremi sono presenti due flangie atte a consentirne il montaggio. Le flange sono saldate mediante tecnologia TIG (Tungsten Inert Gas).

Il componente è realizzato in lega di alluminio $6061 \mathrm{~T} 6$, largamente impiegata nel settore aeronautico, in quanto risulta avere ottime prestazioni in termini di saldabilità, resistenza a corrosione, e caratteristiche meccaniche (vedere Tab. 1). Il disegno dell'albero completo di flangie è rappresentato in Fig. 1.

\begin{tabular}{ccc}
\hline$E[\mathrm{MPa}]$ & Modulo di elasticità longitudinale & 70000 \\
$\nu$ & Coefficiente di Poisson & 0.33 \\
$\varrho\left[\mathrm{Kg} / \mathrm{m}^{3}\right]$ & Massa volumica & 2700 \\
$\sigma_{s n}[\mathrm{MPa}]$ & Carico unitario di snervamento & 289.6 \\
$\sigma_{\mathrm{R}}[\mathrm{MPa}]$ & Carico unitario di rottura & 310 \\
\hline
\end{tabular}

Tabella 1: Proprietà meccaniche della lega Al 6061-T6.

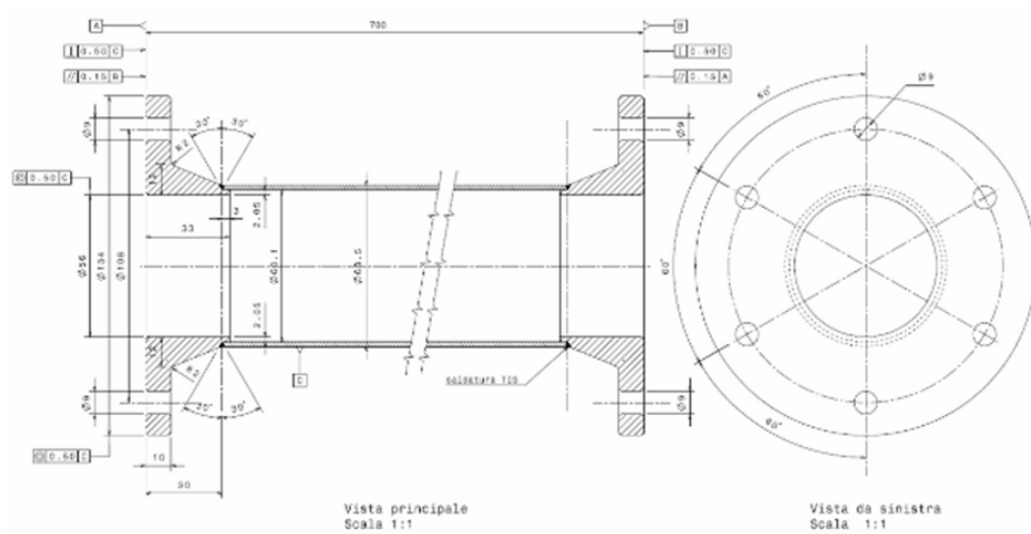

Figura 1: Geometria dell'albero di trasmissione oggetto di studio.

\section{Condirioni di impatto}

I parametri che concorrono alla definizione delle condizioni di impatto, escludendo la velocità del proiettile legata alle caratteristiche dell'arma da fuoco e del proiettile utilizzati, si riducono essenzialmente ad angolo di impatto e all'offset del proiettile, indicati in Fig. 2. Trascurando il caso di impatto con imbardata, l'angolo di impatto rappresenta l'angolo formato dalla traiettoria del proiettile con la retta normale all'asse del bersaglio. Con offset si intende invece la distanza fra l'asse del bersaglio e la traiettoria del proiettile.

Sulla base di numerose simulazioni ad elementi finiti dell'impatto balistico di un proiettile calibro $7.62 \mathrm{NATO}$, contro tubi cilindrici cavi, argomento di precedenti studi [3], è stata individuata la condizione più gravosa in termini di danneggiamento. La pericolosità dell'impatto è stata valutata in base alle dimensioni del foro ottenuto ed all'estensione della zona soggetta a sforzi residui, fattori in grado di influenzare rispettivamente la rigidezza dell'albero e la velocità di propagazione delle cricche nucleate sul bordo del foro. Considerando le dimensioni geometriche esatte dell'albero, la condizione peggiore, indicata in Tab. 2, corrisponde al caso di impatto angolato a $45^{\circ}$ con superficie del proiettile tangente a quella del cilindro (definito caso critico): tale condizione origina un singolo foro di forma pressoché ellittica, con asse inclinato di circa $45^{\circ}$ e di dimensioni massime.

Questa condizione costituisce la tipologia di danneggiamento maggiormente gravosa per un albero di trasmissione sollecitato a torsione. Le simulazioni, condotte variando l'offset del proiettile, hanno mostrato come una riduzione dell'offset rispetto a quello critico provochi la comparsa di due fori distinti di ingresso ed uscita del proiettile; al contrario 
aumentando l'offset oltre il valore critico si genera ancora un unico foro di lunghezza gradualmente inferiore, ottenendo talora il rimbalzo del proiettile (Ricochet effect).

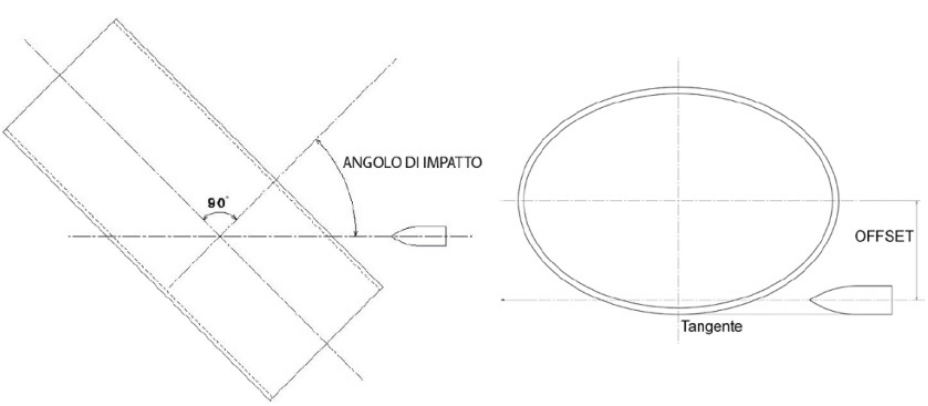

Figura 2: Parametri che definiscono le condizioni di impatto.

\begin{tabular}{cc}
\hline Parametro & $\begin{array}{c}\text { Condizione di massimo } \\
\text { danneggiamento }\end{array}$ \\
\hline Angolo di impatto $\left[{ }^{\circ}\right]$ & 45 \\
Offset del proiettile $[\mathrm{mm}]$ & 28 \\
\hline
\end{tabular}

Tabella 2: Condizioni di impatto.

\section{Set up sperimentale}

Le prove balistiche sono state effettuate presso il poligono di tiro della società Oto Melara (BS). Arma da fuoco, sistema di puntamento e proiettili sono stati forniti dalla stessa società, così come la strumentazione per la misura della velocità iniziale del proiettile.

L'arma da fuoco calibro 7.62 NATO, dotata di puntatore ottico, è montata su un affusto che dispone di due gradi di libertà di rotazione, in un piano verticale ed orizzontale, definiti rispettivamente alzata e brandeggio. Il sistema per la misura della velocità iniziale del proiettile è costituito invece da sorgenti Laser e detector fissati ad un supporto trasportabile. Tutte le altre attrezzature, necessarie per lo svolgimento delle prove, sono state progettate e realizzate nei laboratori del Dipartimento di Meccanica del Politecnico di Milano. La Fig. 3 mostra lo schema del set-up sperimentale utilizzato.

Per il posizionamento degli alberi di trasmissione lungo la linea di tiro, è stato costruito un telaio, utilizzando comuni elementi di carpenteria in acciaio, la cui caratteristica principale è rappresentata dalla possibilità di impostare l'angolo di impatto e l'offset del proiettile. Il provino è libero di traslare orizzontalmente e verticalmente grazie a due afferraggi scorrevoli su una struttura tubolare rettangolare, vincolata a due supporti mediante due perni che permettono di regolarne l'inclinazione. L'utilizzo di giunzioni smontabili ha reso possibile la suddivisione del telaio in vari pezzi di peso ed ingombro limitato, facilmente trasportabili anche a mano.

Come già affermato, il poligono ha fornito i sensori Laser atti alla misura della velocità iniziale del proiettile, mentre posteriormente al provino nessuna strumentazione è stata posizionata a causa dei rischi di danneggiamento connessi ad una possibile deviazione del proiettile.

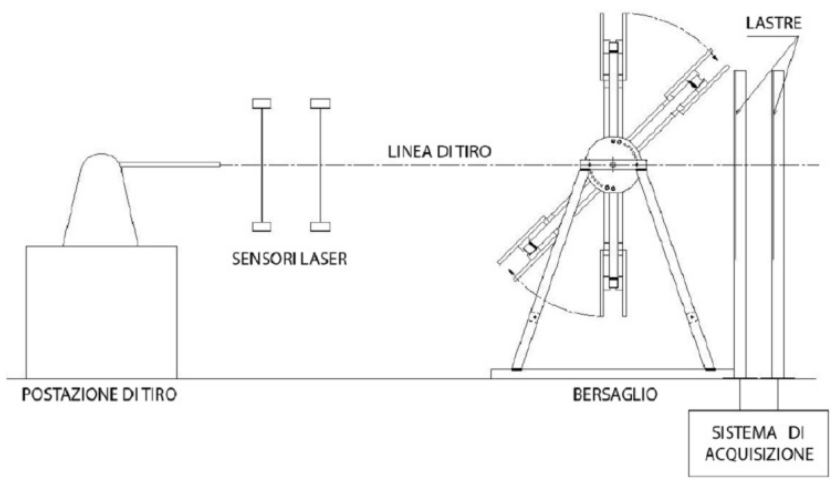

Figura 3: Set-up sperimentale. 
Analizzando le metodologie utilizzate in altre indagini sperimentali [4-15] per la misura della velocità residua del proiettile, è stato sviluppato un sistema basato sull'applicazione di due accelerometri a due lamine sottili di alluminio, definito per chiarezza espositiva metodo delle lastre sacrificali: la perforazione delle due lastre genera due segnali distinti che vengono registrati da un sistema di acquisizione in grado di calcolarne l'intervallo temporale di emissione; nota la distanza fra le lastre si determina facilmente la velocità del proiettile. Per non compiere una sottostima è stata fatta inoltre una valutazione dell'energia cinetica dissipata per la perforazione di una lastra. Il sistema realizzato permette inoltre di stimare la deviazione subita dal proiettile a causa dell'impatto contro il bersaglio, e di valutare la distanza reale percorsa dal proiettile fra le due lastre: ciò può essere compiuto confrontando le posizioni dei fori al termine di ogni test con le posizioni dei fori ottenuti da uno sparo "a vuoto", cioè senza bersaglio montato.

Poiché dopo i primi test sono state osservate deviazioni modeste della traiettoria del proiettile, si è ritenuto vantaggioso, al fine di migliorare l'accuratezza della misura della velocità, sfruttare l'indipendenza dei due telai incrementandone la distanza reciproca.

Analogamente, l'allontanamento della prima lastra dal telaio di supporto degli alberi, ha consentito un più agevole riconoscimento dei fori del proiettile da quelli dovuti all'impatto di eventuali frammenti. La Fig. 4 mostra il banco di sostegno dei provini dietro al quale si trova il sistema per la misura della velocità residua del proiettile. In Fig. 5 è riportata un'immagine del proiettile 7.62 NATO utilizzato.

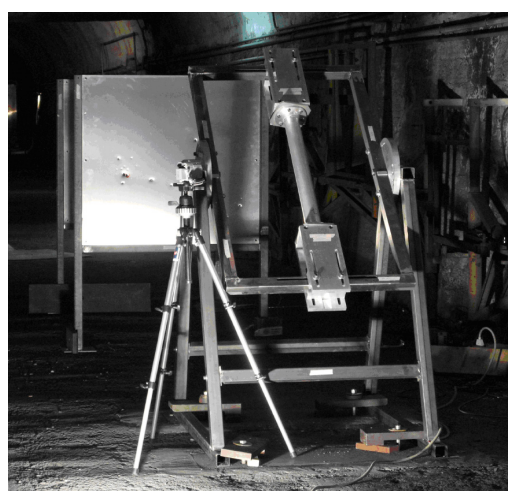

Figura 4: Banco di sostegno e sistema per la misura della velocità residua

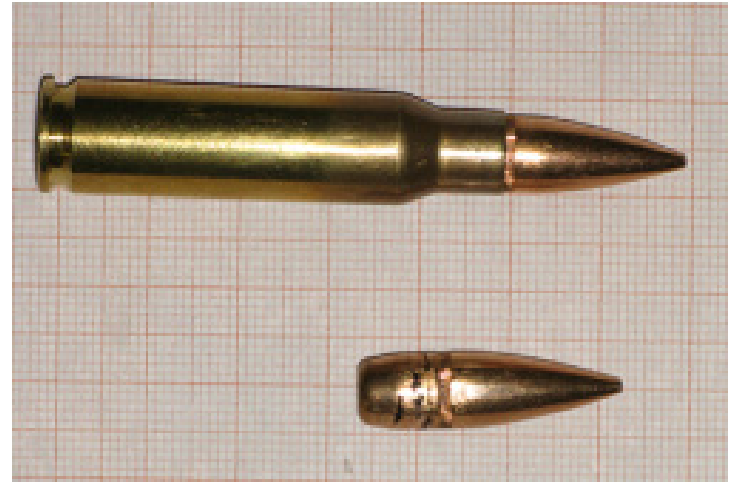

Figura 5: Il proiettile 7.62 NATO; inferiormente solo la "pallottola" estratta dal bossolo.

La strumentazione per l'acquisizione dei segnali è costituita da:

- 2 Accelerometri PCB Piezotronics Model 352C23;

- Amplificatore KISTLER INSTRUMENTS 5122;

- Sistema di acquisizione NI 9215;

- Software LabView 8.0;

Gli accelerometri considerati sono caratterizzati da una banda passante piuttosto ampia: infatti operano correttamente ad una frequenza di $25 \mathrm{KHz}$ anche se la loro risposta è migliore sotto i $10 \mathrm{KHz}$. Tuttavia ciò che interessa in questa applicazione non è la ricostruzione della risposta in frequenza del fenomeno ma semplicemente l'identificazione dell'intervallo temporale fra gli istanti ai quali si ha il contatto del proiettile su ciascuna delle due lastre sacrificali. Il programma Labview ${ }^{\circledR}$ permette di realizzare uno strumento virtuale $(V I)$, in grado di calcolare tale intervallo di tempo.

\section{Risultati delle prove}

L'esecuzione di prove balistiche a vuoto (con colpo non deviato al centro delle lastre) ha permesso di effettuare una validazione del metodo delle lastre sacrificali, confrontando i risultati con quanto rilevato dai laser/detectors.

La corrispondenza fra i risultati appare ottima (si veda la Tab. 3); la lieve sottostima della velocità residua commessa mediante questo metodo, è dovuta al fatto che il proiettile deve attraversare una lastra, seppur sottile, di alluminio; quindi per ridurre ulteriormente l'errore è stata valutata l'energia necessaria per la perforazione di tale lastra, utilizzando la teoria espressa in [16].

La velocità iniziale del proiettile è stata ottenuta attraverso la seguente relazione:

$$
V_{i}=\sqrt{\frac{2\left[E_{C f}+\left(E_{P l u g}+E_{D i s h}+E_{P e t}\right)\right]}{(1-0.02) m_{P}}}
$$


ricavata attraverso il seguente bilancio energetico:

$$
E_{C f}=E_{C i}-\left(E_{P l u g}+E_{\text {Dish }}+E_{P e t}+E_{\text {Attr }}\right)
$$

essendo:

$E_{C f}$ l'energia cinetica finale nota del proiettile dopo la perforazione;

$E_{C i}$ l'energia cinetica iniziale incognita del proiettile;

$E_{\text {Plug }}+E_{\text {Dish }}+E_{P e t}+E_{\text {Attr }}$ l'energia spesa per attraversare una lastra sacrificale, i cui termini si riferiscono rispettivamente al plugging, al dishing, al petaling ed all'attrito.

Dimostrata quindi la validità del metodo delle lastre sacrificali, questo è stato applicato durante i test di impatto per la misura della velocità residua del proiettile.

In questo caso per aumentare l'accuratezza delle misure sono state apportate alcune correzioni che tengono conto non solo dell'energia dissipata dalla perforazione della prima lastra sacrificale, ma anche della traiettoria del proiettile deviato dall'impatto, e del ritardo temporale con cui sono acquisiti i segnali, legato alla velocità di propagazione delle onde all'interno del materiale; in particolare il ritardo sarà differente per i due segnali nel caso in cui il proiettile a causa della deviazione impatti sulle due lastre in posizioni diverse.

\begin{tabular}{cccc}
\hline $\begin{array}{c}\text { Prove } \\
\text { a } \\
\text { vuoto }\end{array}$ & $\begin{array}{c}\text { Laser/Detectors } \\
{[\mathrm{m} / \mathrm{s}]}\end{array}$ & $\begin{array}{c}\text { Lastre Sacrificali } \\
{[\mathrm{m} / \mathrm{s}]}\end{array}$ & $\begin{array}{c}\text { Lastre Sacrificali } \\
\text { corretto } \\
{[\mathrm{m} / \mathrm{s}]}\end{array}$ \\
1 & 839 & 816 & 831 \\
2 & 838 & 825 & 840 \\
3 & 825 & 803 & 817 \\
4 & 832 & 810 & 824 \\
5 & 846 & 826 & 841 \\
6 & 833 & 810 & 824 \\
7 & 855 & 830 & 845 \\
8 & 848 & 830 & 845 \\
9 & 845 & 823 & 838 \\
10 & 840 & 820 & 835 \\
11 & 845 & 823 & 838 \\
12 & 850 & 823 & 838 \\
13 & 854 & 826 & 841 \\
14 & 847 & 826 & 841 \\
15 & 840 & 813 & 828 \\
16 & 834 & 813 & 828 \\
17 & 852 & 826 & 841 \\
18 & 861 & 837 & 852 \\
Media & 844 & 821 & 836 \\
\hline
\end{tabular}

Tabella 3: Velocità del proiettile rilevata negli spari a vuoto; confronto fra i valori forniti dai Laser/Detectors e dalle Lastre Sacrificali.

La Fig. 6 aiuta a comprendere quanto appena esposto. Per la valutazione della traiettoria effettiva del proiettile si sono rilevate le coordinate dei fori sulle due lastre, mentre l'energia assorbita dalla perforazione della prima lastra è stata stimata attraverso il metodo energetico utilizzato in precedenza.

Le velocità iniziale del proiettile, quella residua (corretta e non), e l'angolo di deviazione del proiettile rilevati in tutte le prove di impatto sono contenuti nella Tab. 4.

Dal punto di vista della forma e delle dimensioni del danneggiamento causato dall'impatto balistico, i test sperimentali presentano una dispersione di risultati non trascurabile: in effetti analizzando tali risultati riportati in Tab. 5 è evidente come condizioni di impatto nominalmente identiche portino a risultati macroscopicamente differenti.

La scelta di studiare la condizione più gravosa definita in Tab. 2 ha avuto infatti un peso rilevante nella determinazione di tale disomogeneità: uno scostamento dalle condizioni critiche ricercate, legato alla variabilità sempre presente in qualche misura in un fenomeno di impatto, anche se di piccola entità, può dare origine ad un danneggiamento caratterizzato da un doppio foro, da un singolo foro di varie lunghezza o in certi casi anche al rimbalzo del proiettile. La Fig. 7 mostra le fotografie di due delle principali tipologie di danneggiamento ottenuto. 


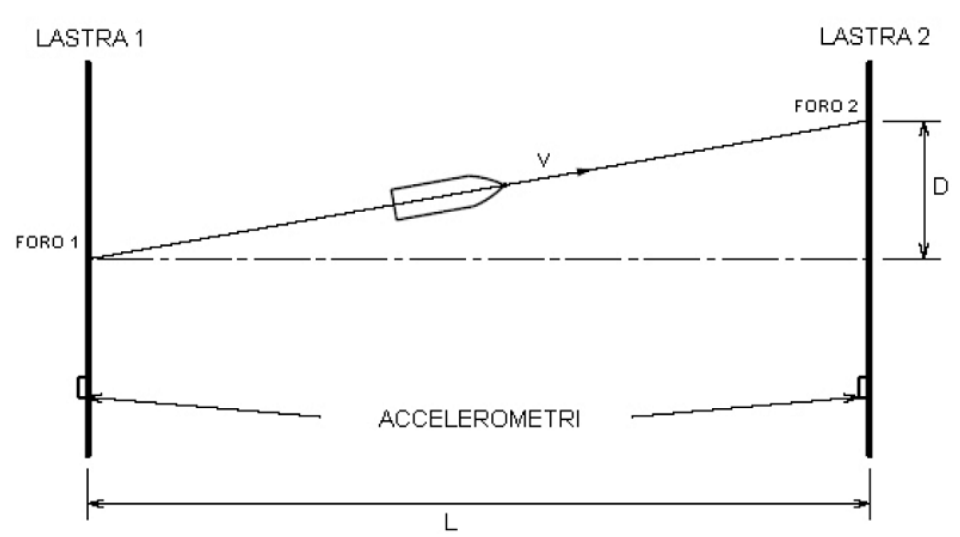

Figura 6: Traiettoria del proiettile deviato dall'impatto: il proiettile percorre uno spazio maggiore della distanza fra le lastre; la distanza D determina un ritardo nell'acquisizione del secondo segnale.

\begin{tabular}{ccccc}
\hline Prova & $V_{i}[\mathrm{~m} / \mathrm{s}]$ & $V_{\mathrm{R}}[\mathrm{m} / \mathrm{s}]$ & $V_{\text {Reff }}[\mathrm{m} / \mathrm{s}]$ & $\alpha\left[^{\circ}\right]$ \\
1 & 859 & 748 & 765 & 1.09 \\
2 & 853 & - & - & 0.94 \\
3 & 853 & 725 & 741 & 1.39 \\
4 & 850 & 714 & 730 & 0.78 \\
5 & 854 & 750 & 767 & 1.15 \\
6 & 847 & 740 & 757 & 0.82 \\
7 & 849 & 724 & 740 & 2.88 \\
8 & 845 & - & - & - \\
9 & 849 & 725 & 741 & 0.52 \\
10 & 859 & 712 & 728 & 2.81 \\
11 & 862 & 714 & 730 & 3.17 \\
12 & 844 & 725 & 741 & 1.90 \\
13 & 855 & 689 & 705 & 1.41 \\
14 & 852 & - & - & - \\
15 & 839 & 709 & 725 & 4.76 \\
16 & 844 & 711 & 727 & 1.25 \\
17 & 848 & 685 & 70 & 3.34 \\
18 & 860 & 754 & 771 & 1.11 \\
Media & 851 & 728 & 744 & 1.83 \\
\hline
\end{tabular}

Tabella 4: Velocità iniziale del proiettile $(\mathrm{V})$, velocità residua $\left(\mathrm{V}_{\mathrm{R}}\right)$, velocità residua effettiva $\left(\mathrm{V}_{\text {Reff }}\right)$ ed angolo di deviazione $(\alpha)$ rilevati in ogni prova sperimentale.

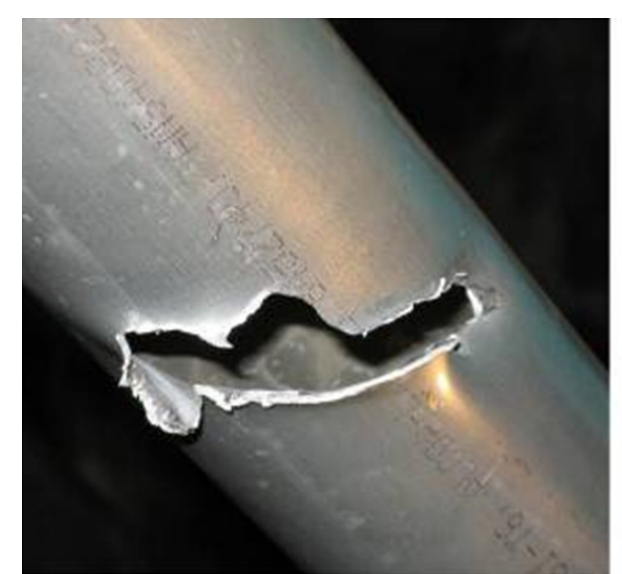

(a)

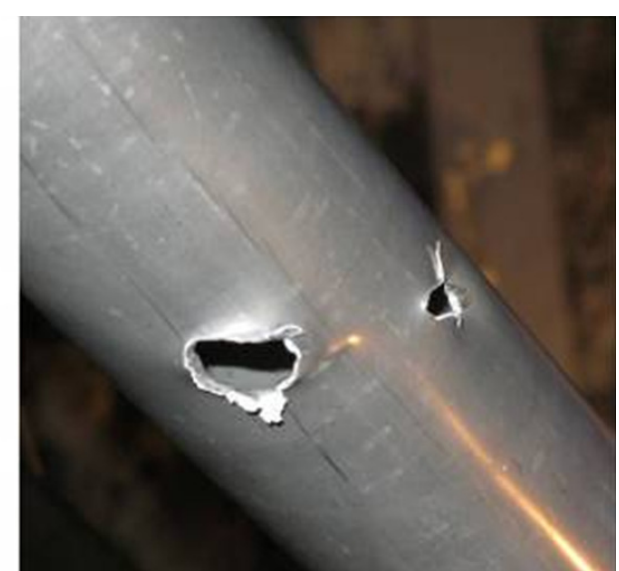

(b)

Figura 7: Principali tipologie di danno balistico osservate al termine dei test: a) singolo foro; b) doppio foro. 
I provini sono quindi stati classificati in base alle dimensioni del danno: per i provini con doppio foro sono state misurate, lungo un'elica cilindrica inclinata a $45^{\circ}$ rispetto all'asse del tubo, la distanza totale fra gli apici dei due fori e la lunghezza del setto di separazione fra di essi; invece per i singoli fori si è rilevata solo la lunghezza totale della lacerazione. Dall'analisi dei risultati ottenuti, si osserva come solamente nel $30 \%$ circa dei casi sia stato ottenuto il danno balistico nominalmente più gravoso.

La Tab. 5 mostra una classificazione degli alberi, effettuata in base alla tipologia di danneggiamento ottenuto, per ognuna delle quali è stato valutato il valore medio delle velocità iniziale e finale del proiettile misurate durante i test: analizzando i dati contenuti nella Tab. 6 si può constatare come la massima riduzione di velocità si sia rilevata nei test che originano un singolo foro a $45^{\circ}$, ai quali è associato il massimo assorbimento energetico.

È stata eseguita infine una valutazione dello stato tensionale residuo superficiale lasciato dall'impatto. Gli sforzi residui sono stati misurati in vari punti su ciascun provino, sia prima che dopo le prove di impatto, utilizzando un analizzatore di tensione a raggi X Stresstech Group modello V.1.01.

La Tab. 7 contiene i valori medi delle tensioni misurate prima e dopo i test.

Le tensioni rilevate dopo l'impatto ad una distanza massima di $20 \mathrm{~mm}$ dagli apici del foro, mostrano in generale andamenti differenti considerando le due zone adiacenti al foro di ingresso e di uscita del proiettile, risultando prevalentemente di trazione nella prima e di compressione nella seconda.

\begin{tabular}{cccccc}
\hline TIPO DI D ANNO & LTOT $[\mathrm{mm}]_{\text {L }}$ & SETTO $_{\mathrm{mm}]}$ & L FORI $_{\mathrm{mm}]}$ & $N^{\circ}$ Provini \\
Singolo foro a $45^{\circ}$ & da 75 a 85 & - & - & $10,11,13,14,17$ \\
& da 75 a 85 & fino a 20 & da 30 a 40 & $1,2,3$ \\
Doppio foro a $45^{\circ}$ & da 75 a 85 & da 20 a 35 & da 20 a 30 & $5,6,16$ \\
& da 80 a 90 & da 35 a 50 & da 15 a 25 & $4,7,9,12$ \\
Singolo foro imbozzato & 45 & - & - & 8 \\
Impatto di striscio & 21 & - & - & 15 \\
Doppio foro a $0^{\circ}$ & 90 & 69 & da 10 a 11 & 18 \\
\hline
\end{tabular}

Tabella 5: Classificazione dei provini in base alla tipologia di danneggiamento.

\begin{tabular}{|c|c|c|c|c|c|}
\hline \multicolumn{2}{|c|}{ CLASSE DI DANNO } & $V_{i}[\mathrm{~m} / \mathrm{s}]$ & $V_{f}[\mathrm{~m} / \mathrm{s}]$ & $V_{\text {feff }}[\mathrm{m} / \mathrm{s}]$ & $\Delta V / V_{i}[\%]$ \\
\hline \multicolumn{2}{|c|}{ Intaglio singolo a $45^{\circ}$} & 856 & 700 & 713 & $-16,7$ \\
\hline \multirow{3}{*}{ Doppio foro a $45^{\circ}$} & setto piccolo & 855 & 737 & 750 & $-12,3$ \\
\hline & setto medio & 848 & 734 & 747 & $-11,9$ \\
\hline & setto grande & 848 & 722 & 735 & $-13,2$ \\
\hline \multicolumn{2}{|c|}{ Impatto di striscio a $45^{\circ}$} & 839 & 709 & 722 & $-13,9$ \\
\hline \multicolumn{2}{|c|}{ Doppio foro a $90^{\circ}$} & 860 & 754 & 768 & $-10,7$ \\
\hline \multicolumn{2}{|c|}{ Valore medio } & 851 & 728 & 741 & $-12,9$ \\
\hline
\end{tabular}

Tabella 6: Valori sperimentali medi della velocità incidente e residua effettiva del proiettile relativi alle varie classi di danneggiamento.

\begin{tabular}{cccccc}
\hline \multicolumn{2}{c}{} & \multicolumn{4}{c}{ Dopo l'impatto } \\
Prima dell'impatto & \multicolumn{3}{c}{ IN } & \multicolumn{2}{c}{ OUT } \\
\hline & & $\sigma_{I}[\mathrm{MPa}]$ & $\sigma_{I I}[\mathrm{MPa}]$ & $\sigma_{I}[\mathrm{MPa}]$ & $\sigma_{I I}[\mathrm{MPa}]$ \\
89,33 & $\sigma_{I I}[\mathrm{MPa}]$ & $\sigma_{I}[14,04$ & 7,37 & 3,97 & $-128,40$ \\
\hline
\end{tabular}

Tabella 7: Valore medio delle tensioni residue rilevate prima e dopo le prove di impatto balistico; dopo l'impatto sono state considerate separatamente le zone di ingresso e di uscita del proiettile per evidenziare il differente comportamento osservato nelle due aree 


\section{PROVE TORSIONALI SIMULANTI I CARICHI DELLA MISSIONE DI RIENTRO}

\section{Misure effettuate e metodi}

$\mathrm{D}$

urante le prove torsionali, che costituiscono l'ultima fase della sperimentazione effettuata, sono state misurate le deformazioni applicando rosette estensimetriche a tre griglie a $0^{\circ}, 45^{\circ}$ e $90^{\circ}$, con compensazione termica, il cui impiego è dettato dalla possibilità di determinare lo stato di sollecitazione principale incognito conoscendo le deformazioni lungo tre direzioni note.

Sforzi e direzioni principali possono essere calcolati risolvendo il seguente sistema di 3 equazioni nelle 3 incognite $\sigma_{I}$, $\sigma_{I I}$ e $\theta$ :

$$
\begin{aligned}
& \sigma_{I, I I}=\frac{E}{2(1-v)}\left(\varepsilon_{a}+\varepsilon_{c}\right) \pm \frac{E}{\sqrt{2}(1+v)} \sqrt{\left(\varepsilon_{a}-\varepsilon_{b}\right)^{2}+\left(\varepsilon_{c}-\varepsilon_{b}\right)^{2}} \\
& \operatorname{tg} 2 \theta=\frac{2 \varepsilon_{b}-\varepsilon_{a}-\varepsilon_{c}}{\varepsilon_{a}-\varepsilon_{c}}
\end{aligned}
$$

Per acquisire i segnali estensimetrici è stata utilizzata la seguente attrezzatura:

- Chassis National Instrument SCXI-1000;

- 3 moduli di acquisizione National Instrument SCXI-1121 a 4 canali;

- 3 moduli estensimetrici National Instrument SCXI-1321 a 4 canali;

- Software di acquisizione dedicato;

Le rosette sono state applicate sui provini nella zona adiacente al danno balistico, come visibile nella Fig. 8.
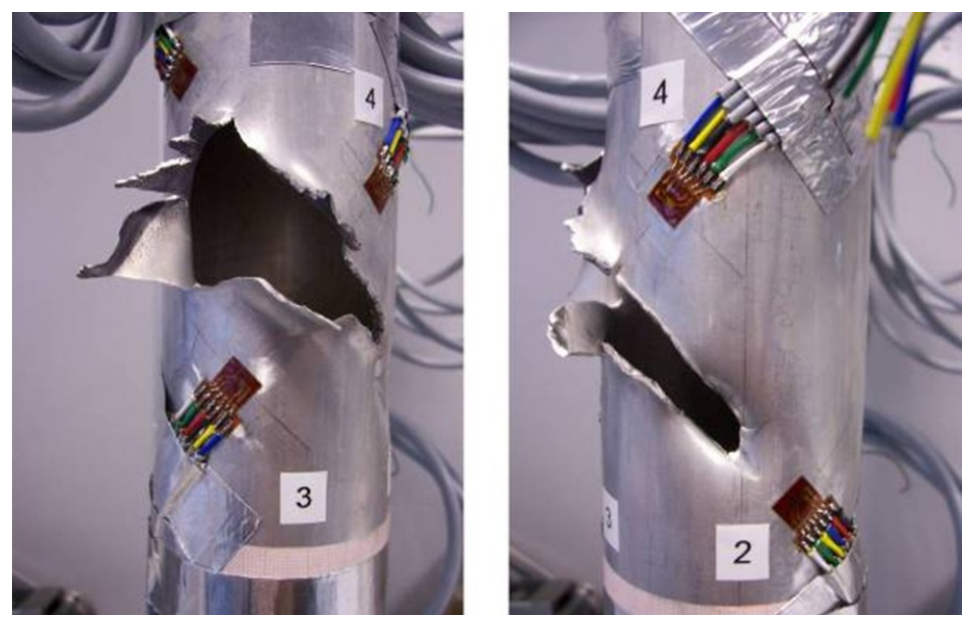

Figura 8: Posizione delle rosette.

Durante le prove di fatica torsionale è stata inoltre monitorata la propagazione delle cricche, osservate mediante un microscopio ottico, dotato di hardware e software per l'acquisizione di immagini.

Nella Fig. 9 sono visibili le fotografie ingrandite di una cricca, mentre le Fig. 10 e 11 mostrano rispettivamente la strumentazione utilizzata per monitorare le cricche ed il particolare della zona monitorata di un provino.
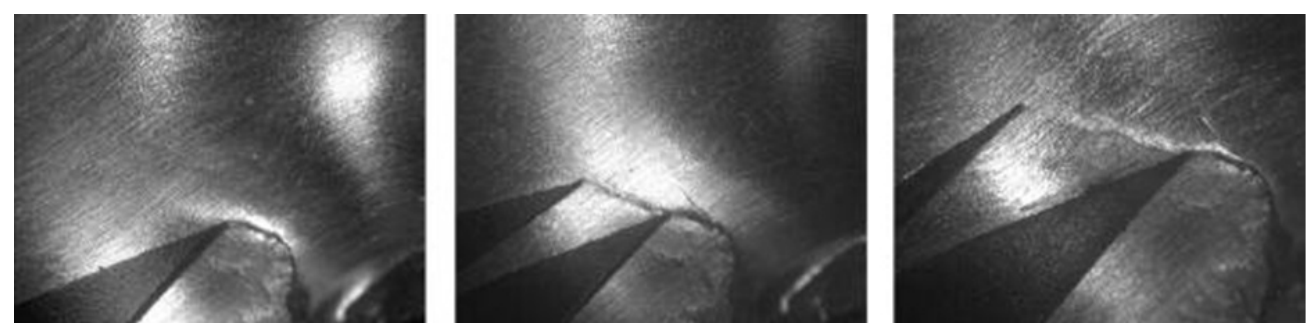

Figura 9: Fotografie ingrandite di una cricca. 


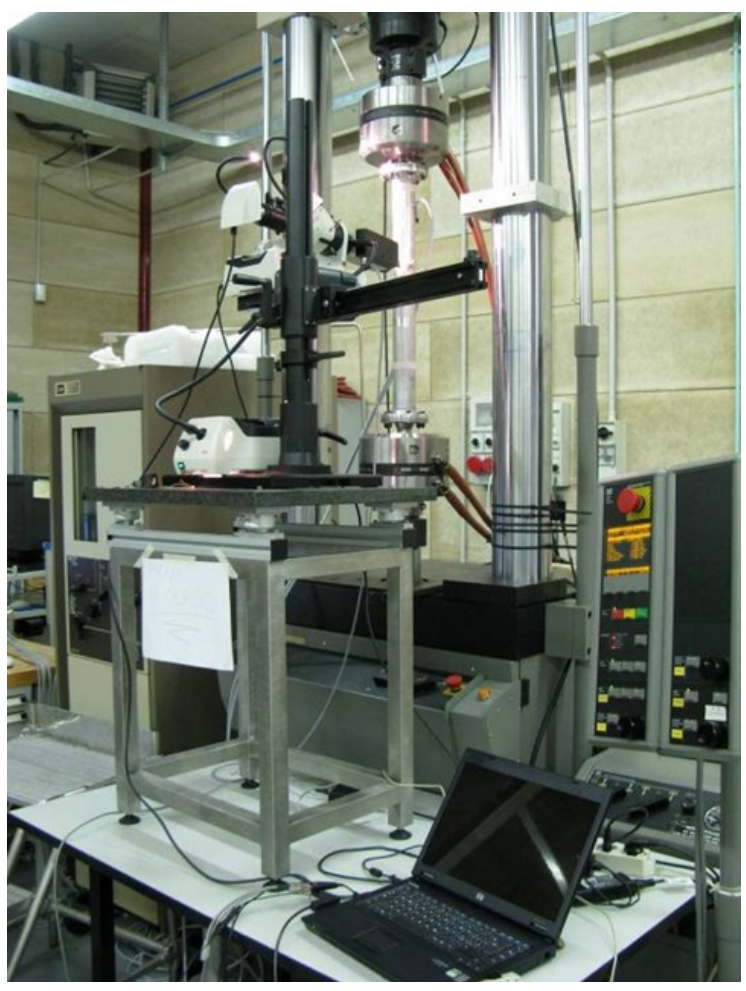

Figura 10: Strumentazione utilizzata per monitorare l'avanzamento delle cricche.

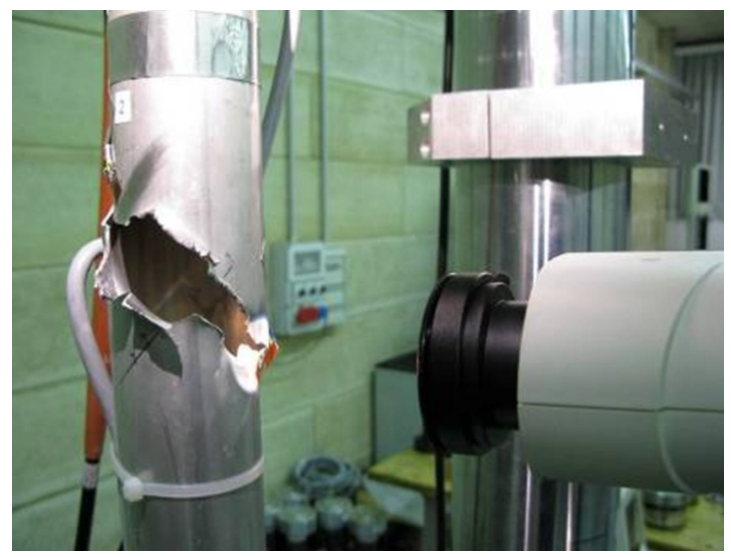

Figura 11: Fotografia di un albero danneggiato dopo l'impatto balistico.

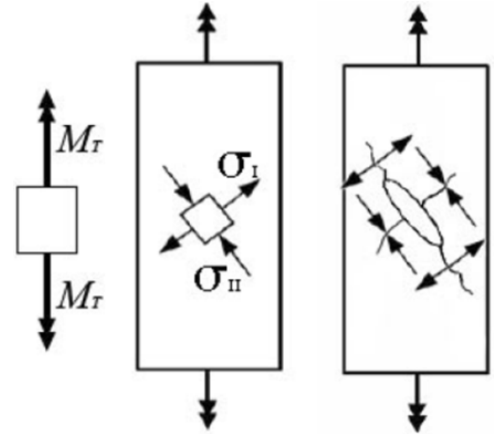

Figura 12: Direzione della coppia applicata

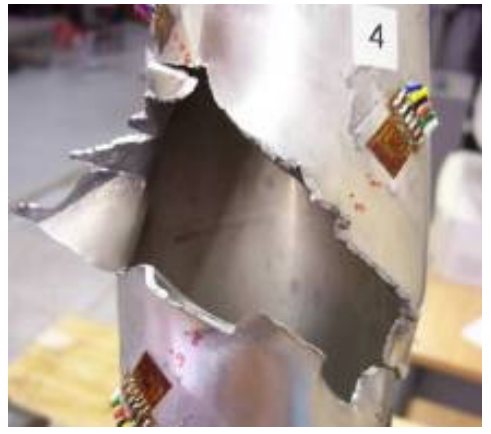

Figura 13: Fotografia del provino danneggiato al termine della prova statica. 
La rottura nel test effettuato sul provino danneggiato dall'impatto balistico si è verificata a partire dagli apici del foro, lungo un'elica cilindrica inclinata a $45^{\circ}$ rispetto all'asse del provino, come visibile nella Fig. 13.

I risultati di entrambe le prove sono rappresentati nella Fig. 14, dalla quale si nota una notevole riduzione della coppia di rottura dell'albero danneggiato rispetto a quello integro.

Tale differenza sarebbe in realtà ancora superiore dal momento che la rottura sul componente integro si è verificata lungo uno dei due cordoni di saldatura in corrispondenza di una coppia pari a $1360 \mathrm{Nm}$ e dunque inferiore al momento di plasticizzazione totale teorico dell'albero pari all'incirca a $1650 \mathrm{Nm}$.

Come già affermato sono stati valutati gli sforzi e le direzioni principali calcolati a partire dalle deformazioni rilevate da ciascuna rosetta applicata sul provino danneggiato.

Elaborando i segnali estensimetrici acquisiti si vede, come era lecito attendersi, che la sollecitazione massima si ha in prossimità degli apici del difetto, in cui sono stati rilevati gli sforzi principali più elevati, rappresentati dalle curve blu e viola nelle Fig. 15 e 16 e riferiti a rosette posizionate come in Fig. 17.

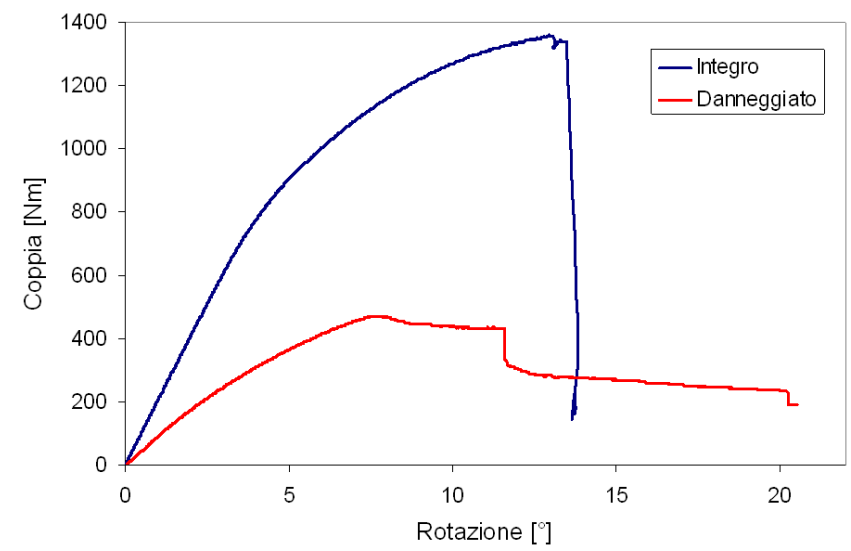

Figura 14: Confronto fra le curve coppia-rotazione ottenute dalle prove effettuate su un provino integro e su un provino con danneggiamento balistico

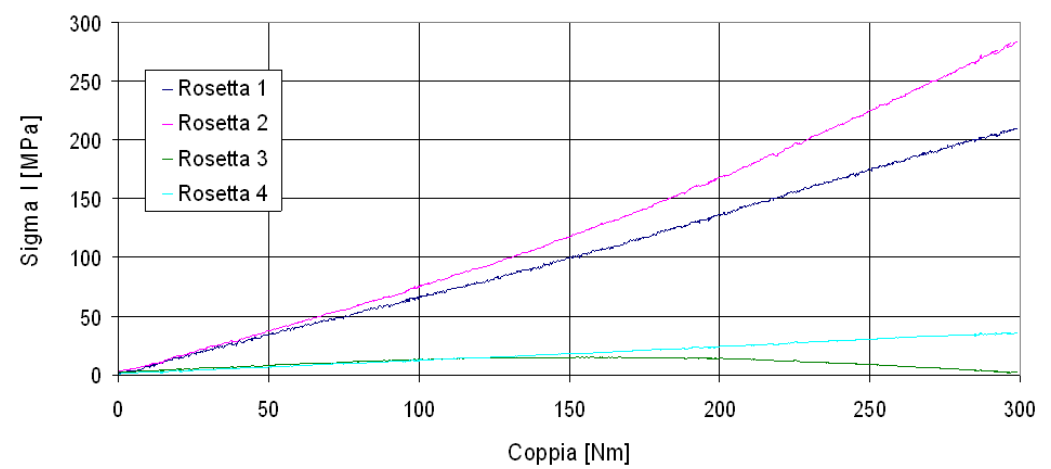

Figura 15: Andamento dello sforzo principale $\sigma_{\mathrm{I}}$ ricavato sulla base dei dati estensimetrici durante la prova statica.

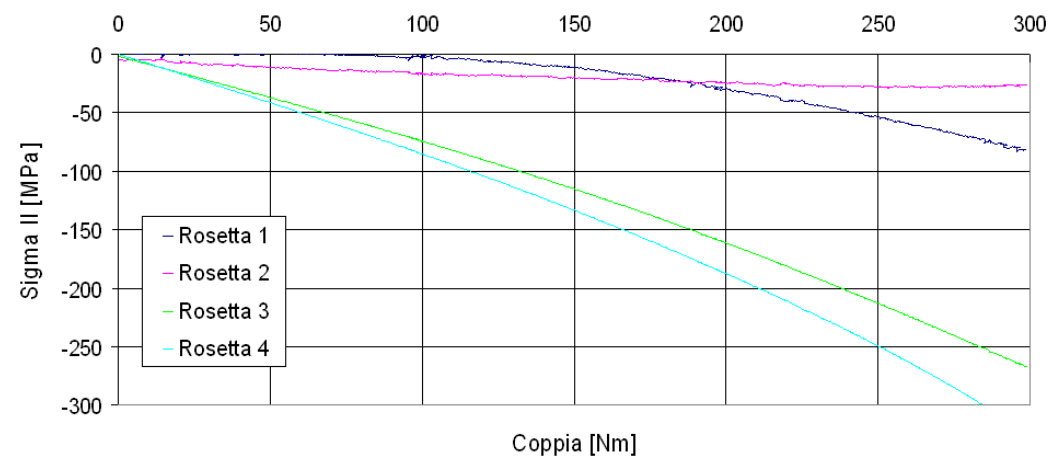

Figura 16: Andamento dello sforzo principale $\sigma_{\text {II }}$ durante la prova statica. 


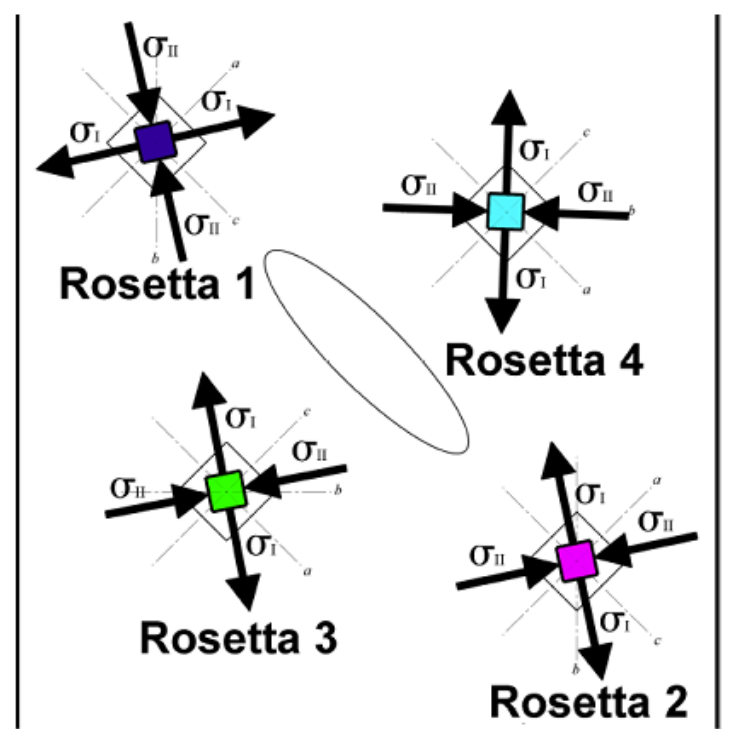

Figura 17: Direzioni principali degli sforzi rilevati durante la prova statica. Il proiettile è entrato nel componente in prossimità della rosetta 2 ed è uscito in prossimità della rosetta 1.

\section{Applicarione degli spettri di carico che simulano la missione di rientro alla base}

Lo scopo di tutta la sperimentazione è verificare che l'elicottero sia in grado di portare a termine una missione di rientro alla base a potenza ridotta, con un albero di trasmissione del rotore di coda danneggiato dall'impatto di un proiettile. Ciò si traduce nella verifica della capacità del componente danneggiato di resistere alle sollecitazioni per tutta la durata della missione.

Da un punto di vista operativo si tratta dunque di applicare all'albero di trasmissione opportuni spettri rappresentativi dei carichi torsionali di manovra (indicati nella Tab. 8), e verificare che non si abbia la rottura del componente.

In questa sede sono mostrati i risultati di una prova rappresentativa condotta su un provino danneggiato con singolo foro (il caso più gravoso). Anche in questo caso, nella zona maggiormente sollecitata, prossima agli apici del difetto, sono state applicate due rosette (si veda la Fig. 18).

Le Fig. 19 e 20 mostrano l'andamento degli sforzi principali $\sigma_{I}$ e $\sigma_{I I}$ rilevati dalle due rosette durante tutta la prova: in ogni grafico le curve rosse e viola rappresentano il massimo e il minimo dello sforzo $\sigma_{I}$ mentre le curve blu e azzurro il massimo e minimo di $\sigma_{I I}$.

\begin{tabular}{ccccc}
\hline SPETTRO & $M_{\text {T Medio }}[\mathrm{Nm}]$ & $M_{\text {T Ampiezia }}[\mathrm{Nm}]$ & Cicli & $\mathrm{R}$ \\
1 & 208 & 48 & 2200 & 0,63 \\
2 & 38 & 28 & 4200 & 0,15 \\
3 & 30 & 24 & 160000 & 0,11 \\
\hline
\end{tabular}

Tabella 8: Spettri di carico rappresentativi delle manovre effettuate in una missione di rientro.
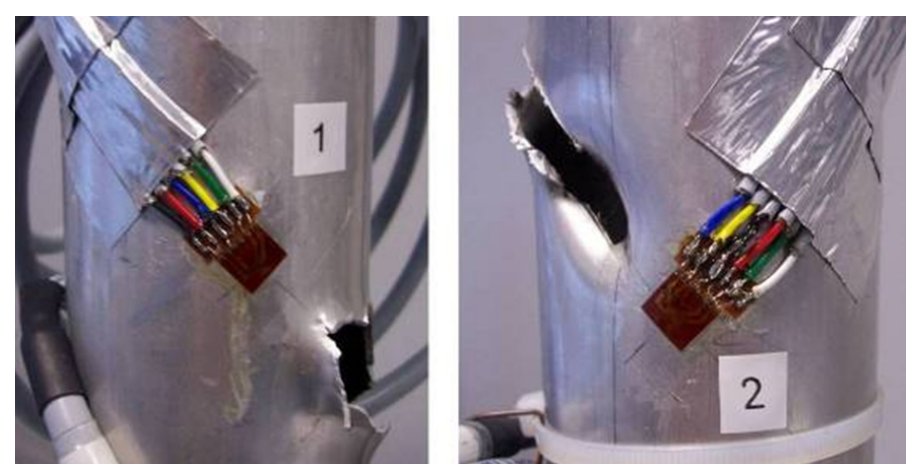

Figura 18: Posizione delle rosette. 
Sforzi Principali Rosetta 1

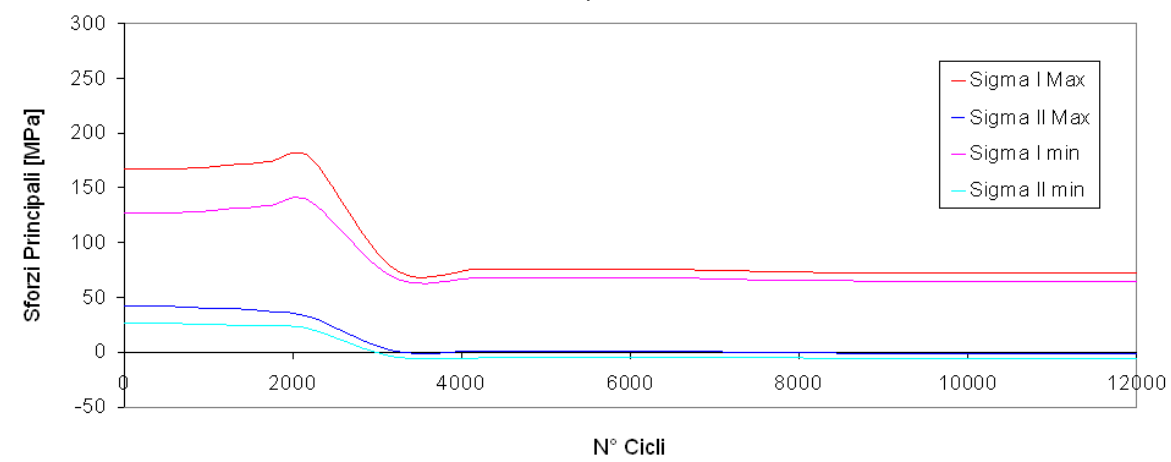

Figura 19: Andamento degli sforzi principali rilevati dalla Rosetta 1 in funzione del $\mathrm{N}^{\circ}$ di cicli: le curve di colore rosso/viola e $\mathrm{blu} /$ azzurro indicano rispettivamente i valori massimo/minimo degli sforzi principali $\sigma_{\mathrm{I}}$ e $\sigma_{\mathrm{II}}$

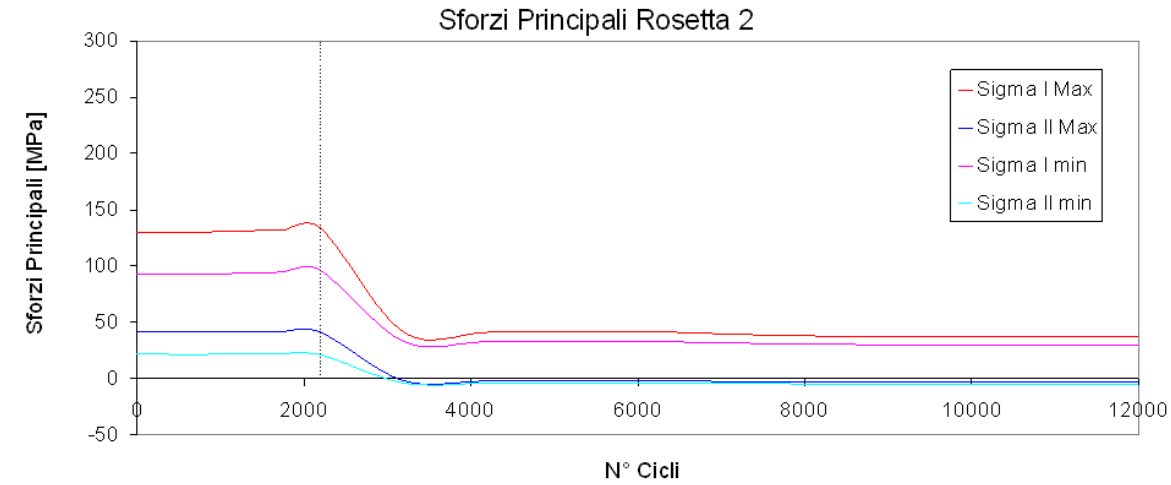

Figura 20: Andamento degli sforzi principali rilevati dalla rosetta 2 in funzione del $\mathrm{N}^{\circ}$ di cicli: le curve di colore rosso/viola e $\mathrm{blu} /$ azzurro indicano rispettivamente i valori massimo/minimo degli sforzi principali $\sigma_{\mathrm{I}}$ e $\sigma_{\mathrm{II}}$.

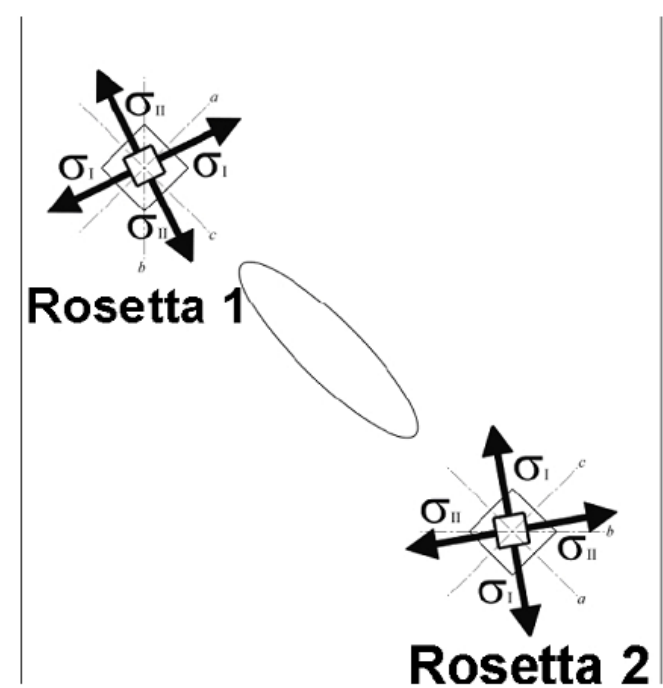

Figura 21: Andamento delle direzioni principali rilevate dalle due rosette.

Durante la prova, in corrispondenza dell'apice di uscita del proiettile, è stata osservata la formazione di una cricca, il cui avanzamento in funzione del numero di cicli è riportato nella Fig. 22: la cricca si è propagata in modo stabile solo durante l'applicazione del primo spettro di carico, contraddistinto da sollecitazioni più elevate, arrestandosi invece nel corso degli altri due spettri.

L'esecuzione di altre prove su alberi di trasmissione con stessa tipologia di danneggiamento ha fornito risultati del tutto analoghi. 
Possiamo dunque affermare che la verifica di resistenza per l'albero danneggiato analizzato è soddisfatta, compatibilmente con la missione definita in precedenza. Ne consegue che l'elicottero sarà in grado di portare a termine con successo la missione di rientro alla base in regime di potenza ridotta.

\section{Lunghezza della Cricca}

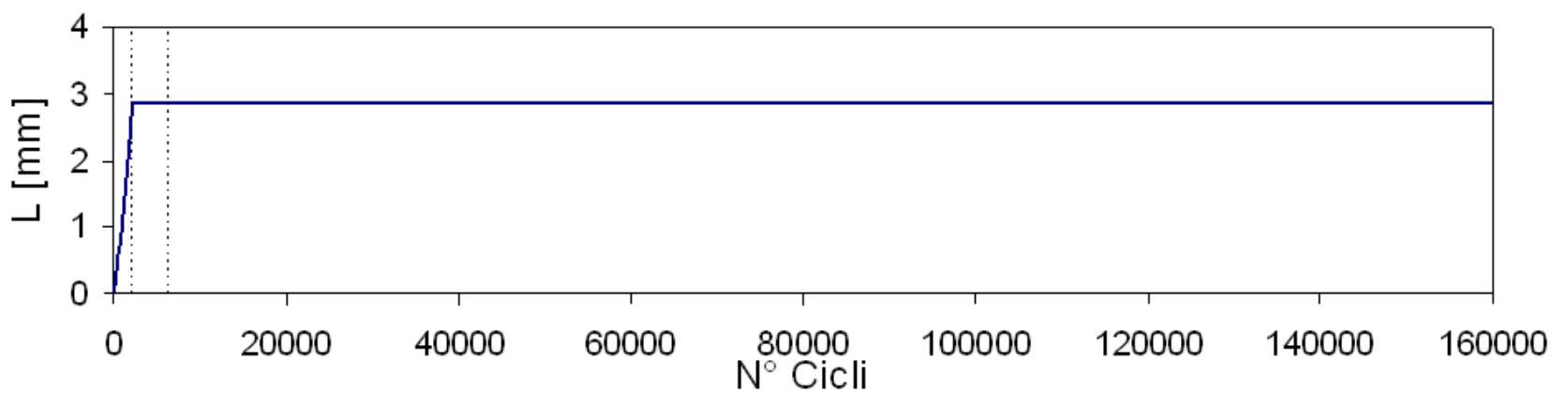

Figura 22: Lunghezza della cricca rilevata in funzione del $\mathrm{N}^{\circ}$ di cicli.

\section{Definizione di una condizione di propagazione instabile}

Accanto alle prove sperimentali che simulano la missione di rientro sono state effettuate altre due prove con lo scopo di definire degli spettri di carico in grado di determinare una condizione di propagazione instabile con conseguente rottura del componente. Dall'analisi dei momenti applicati durante le prove effettuate è stato elaborato un secondo set di carichi (indicato nella Tab. 9) ricavato dal primo spettro (spettro 1) del set precedente, mantenendo invariate coppia massima $\mathrm{e}$ numero di cicli, ed operando una progressiva riduzione del rapporto di carico $\mathrm{R}$, alla quale consegue un aumento del $\Delta \mathrm{K}$ e una maggiore velocità di propagazione della cricca.

L'avanzamento della cricca rilevato nel corso di una delle due prove è rappresentato nella Fig. 23.

La Fig. 24 mostra invece l'andamento degli sforzi principali rilevati dalla rosetta applicata in prossimità dell'apice del danneggiamento da cui si è propagata la cricca.

Nel corso della prova la cricca è avanzata dapprima stabilmente raggiungendo durante l'applicazione dello spettro 3 la condizione di propagazione instabile, a cui è seguito istantaneamente il collasso strutturale del componente.

\begin{tabular}{cccccc}
\hline SPETTRO & $M_{\text {T Med }}[\mathrm{Nm}]$ & $M_{\text {T Amp }}[\mathrm{Nm}]$ & $M_{\text {TMax }}[\mathrm{Nm}]$ & CICLI & $\mathrm{R}$ \\
1 & 208 & 48 & 256 & 2200 & 0,63 \\
2 & 166 & 90 & 256 & 2200 & 0,30 \\
3 & 141 & 115 & 256 & 2200 & 0,10 \\
\hline
\end{tabular}

Tabella 9: Set di carichi derivati dallo spettro $n^{\circ} 1$ del set precedente mantenendo invariate coppia massima applicata e numero di cicli e riducendo progressivamente il rapporto di carico $\mathrm{R}$.

\section{Lunghezza della Cricca}

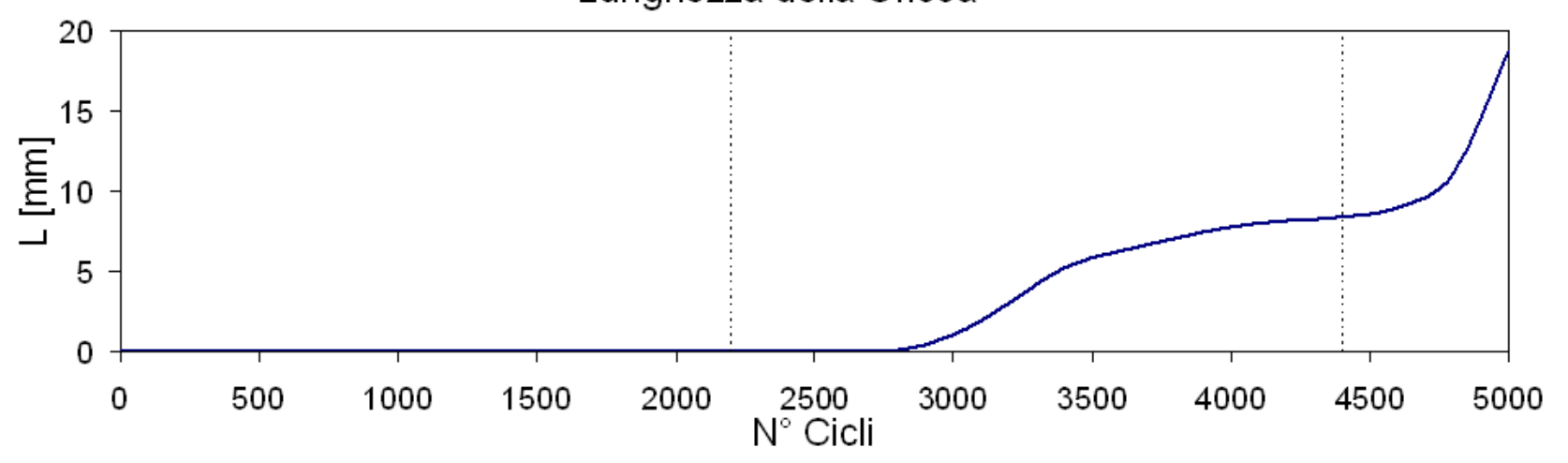

Figura 23: Lunghezza della cricca rilevata nel primo test in funzione del $n^{\circ}$ di cicli: la velocità di propagazione aumenta improvvisamente nel corso del $3^{\circ}$ spettro cui corrisponde il minimo rapporto R. 


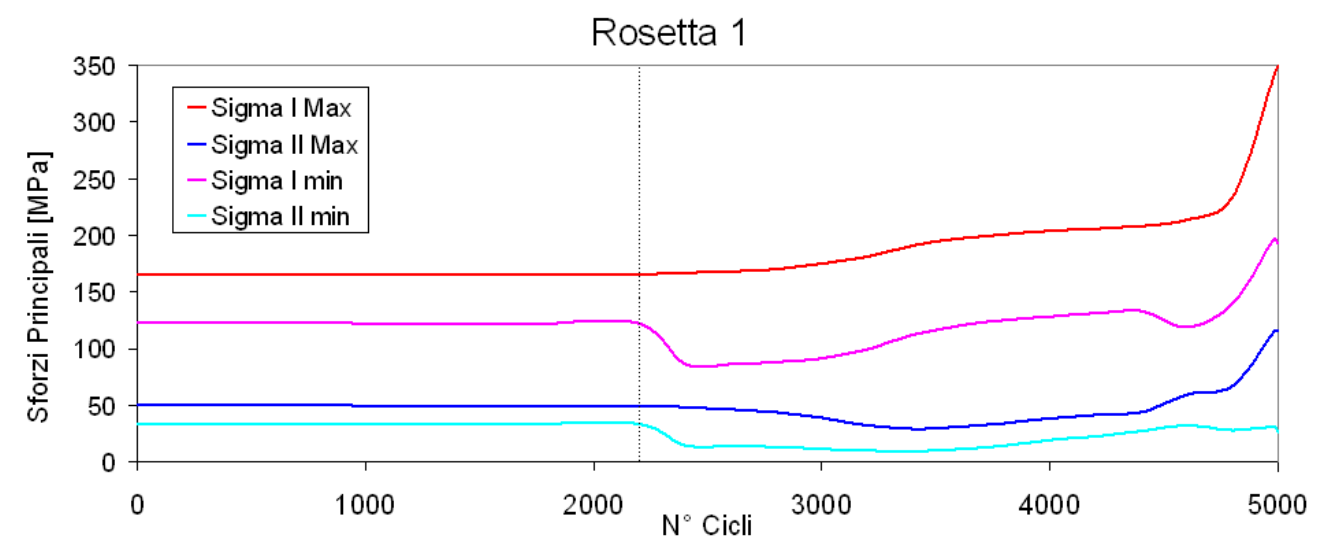

Figura 24: Andamento degli sforzi principali in funzione del $\mathrm{n}^{\circ}$ di cicli ricavati dalle deformazioni misurate durante il primo test in prossimità dell'apice criccato del danneggiamento: si nota un notevole incremento dello sforzo durante il $3^{\circ}$ spettro caratterizzato dal minimo rapporto $\mathrm{R}$

\section{CONCLUSIONI}

$\mathrm{S}$ ono state effettuate alcune prove di impatto balistico, condotte utilizzando un proiettile $7.62 \mathrm{NATO}$, sparato contro provini rappresentativi di un albero di trasmissione sotto ben precise condizioni. I test eseguiti hanno mostrato una dispersione di risultati piuttosto marcata, attribuibile all'estrema criticità che caratterizza il posizionamento reciproco bersaglio-proiettile e ad altri parametri legati alla balistica esterna che sono oggetto di approfondimento attuale.

Agli alberi danneggiati sono quindi stati applicati degli spettri di carico rappresentativi delle sollecitazioni riscontrate durante la missione di rientro alla base. Al fine di contenere la variabilità dei risultati si è testato un campione di provini il più possibile omogenei fra loro e con un danneggiamento di tipo "singolo foro", che è stato considerato come quello di maggiore pericolosità per un componente sottoposto a carichi torsionali.

Le prove di torsione effettuate hanno fornito in effetti esiti analoghi, dai quali si può concludere che l'elicottero, benché presenti un componente principale della linea di trasmissione di coda danneggiato da un proiettile, è in grado di portare a termine una missione di rientro alla base a potenza ridotta, dal punto di vista della resistenza strutturale. In relazione agli obiettivi prefissi, la verifica può quindi considerarsi soddisfatta.

Partendo dagli spettri applicati, e riducendo progressivamente il rapporto di carico $\mathrm{R}$ a parità di coppia massima, è stata definita inoltre una condizione di carico più gravosa, in grado di determinare la propagazione instabile della frattura e dunque il collasso strutturale dell'albero.

Dall'esecuzione di questa seconda serie di test è emerso come il rapporto di carico $\mathrm{R}$ rivesta un ruolo decisivo nel determinare la vita residua a fatica del componente.

\section{BIBLIOGRAFIA}

[1] M. Backmann, W. Goldsmith, Int Engng Sci, (1978).

[2] W. Goldsmith, Int. J. Impact Engng, (1999).

[3] D. Colombo, Progettazione di componenti di elicottero in presenza di cricche e difetti. Tesi di dottorato, Politecnico di Milano, Dipartimento di Meccanica, (2005).

[4] M.J. Forrestal, A.J. Piekutowsky, Int. J. Impact Engng, (1999).

[5] A.J. Piekutowsky, M.J. Forrestal, K.L. Poormon, T.L. Warren, Int. J. Impact Engng, (1999).

[6] T.L. Warren, K. L. Poormon, Int. J. Impact Engng, (2001).

[7] I.V. Roisman, K. Weber, A.L. Yarin, V. Hohler, M.B. Rubin, Int. J. Impact Engng, (1999).

[8] S. Dey, T. Borvik, O. S. Hopperstad, J. R. Leinum., M. Langseth, Int. J. Impact Engng, (2004).

[9] T. Borvik, O.S. Hopperstad, M. Langseth, K. A. Malo, Int. J. Impact Engng, (2002).

[10] T. Borvik, M. Langseth, O.S. Hopperstad, K.A. Malo, Int. J. Impact Engng, (2000).

[11] T. Borvik, M. Langseth, O.S. Hopperstad, K. A. Malo, Int. J. Impact Engng, (1999).

[12] N. K. Gupta, M. A. Iqbal, G. S. Sekhon, Int. J. Impact Engng, (2005). 
[13] M. Zeinoddini, G. A. R. Parke, J. E. Harding, Int. J. Impact Engng, (2000).

[14] N. K. Gupta, V. Madhu, Int. J. Impact Engng, (1997).

[15] L. Kezhun, W. Goldsmith, Int. J. Impact Engng, (1995).

[16] J. Ning, W. Song, J. Wang, Acta Mech. Sinica, (2005). 